\title{
Resections for colorectal liver metastasis: the breakthrough of laparoscopic surgery?
}

\author{
Mohammad Abu Hilal ${ }^{1,2}$, Christoph Kuemmerli ${ }^{1,2}$ \\ ${ }^{1}$ Department of Surgery, Istituto Fondazione Poliambulanza, Brescia, Italy; ${ }^{2}$ Department of Surgery, University Hospital Southampton NHS \\ Foundation Trust, Southampton, UK \\ Correspondence to: Mohammad Abu Hilal, MD, FRCS, FACS, EBSQ (HPB), PhD, DocEurp. Professor, Department of Surgery, Istituto Fondazione \\ Poliambulanza, Via Bissolati 57, 25124 Brescia, Italy. Email: abuhilal9@gmail.com. \\ Provenance and Peer Review: This article was commissioned by the editorial office, Hepatobiliary Surgery and Nutrition. The article did not undergo \\ external peer review. \\ Comment on: Syn NL, Kabir T, Koh YX, et al. Survival advantage of laparoscopic versus open resection for colorectal liver metastases: a meta-analysis \\ of individual patient data from randomized trials and propensity-score matched studies. Ann Surg 2019. [Epub ahead of print].
}

Submitted Feb 26, 2020. Accepted for publication Mar 26, 2020.

doi: $10.21037 /$ hbsn.2020.04.03

View this article at: http://dx.doi.org/10.21037/hbsn.2020.04.03

Liver resection is still considered the treatment of choice for colorectal liver metastasis (CRLMs) with 5 -year survival rates of $40-58 \%$ when associated with modern regimes of chemotherapy. Traditionally, liver resections have been performed through open surgical access. In the last two decades, laparoscopic liver surgery (LLS) has been gaining acceptance with many studies confirming its feasibility, safety and oncological efficiency. Improvement in outcomes have partially been explained by the advantages of minor surgical trauma when maintaining the integrity of the abdominal wall and by reducing intraabdominal scarring. Moreover, a number of techniques have been developed and implemented to ensure that parenchymal-preserving resections can be safely performed in any segment with comparable results to those seen in open surgery $(1,2)$. The feasibility of such oncological resections in the context of a minimally invasive approach has certainly led to many specific advantages especially when dealing with CRLMs, encouraging the feasibility of repeated hepatectomies (3). This is obviously a huge advantage in metastatic colorectal cancer, nowadays considered as a chronic systemic disease, with high incidence of recurrence. Hence, creating optimal conditions for reinterventions is paramount and should be a mainstay of the surgical approach to CRLMs.

The Southampton Consensus Guidelines for Laparoscopic Liver Surgery has confirmed that laparoscopy is a valid approach to treat CRLMs in any location of the liver. In addition, its advantage in repeat hepatectomies was shown (4). The focus has then moved towards long-term oncologic outcome.

The impact of repeat resections facilitated by the adoption of a minimally invasive approach on oncological long-term outcomes has not yet been fully assessed. Interestingly, a recent multicenter study has shown a similar disease-free survival (DFS) but a better overall survival (OS) in patients treated laparoscopically when compared to those treated with open surgery in case of positive resection margins (5). The only difference between the two groups was a higher incidence of redo surgery in patients undergoing LLS. More data, from large cohorts and longer follow ups on this topic are seriously needed.

The first randomized controlled trial (OSLO-COMET) has now been published. It showed that patients undergoing parenchymal-sparing laparoscopic liver resection for CRLMs had a similar median OS and recurrencefree survival to open liver resection (6). The second trial (LapOpHuva) has demonstrated similar oncologic outcomes, with no difference in 5-year DFS or OS between the minimally invasive and the open arm (7). Due to the long recruitment period of 11 years, long-term results were readily available.

Syn et al. published a systematic review on laparoscopic and open surgery for CRLMs focusing on OS (8). Strict in- and exclusion criteria selected relatively high-quality 
studies including the two RCTs. In the pooled cohort with 3,148 patients, LLS was associated with a lower hazard of death (hazard ratio: 0.853, 95\% CI: 0.754-0.965, $\mathrm{P}=0.0114)$. Moreover, the authors also estimate a fraction of patients that can be cured or are long-term survivors, defined as no recurrence during a follow $>10$ years. The cure rates were $47.4 \%$ and $18.0 \%$ in the laparoscopic and open group, respectively, and the mean survival time was longer in the laparoscopic group. In a subgroup of patients with synchronous resections of the primary tumor and CRLMs no survival advantage of the laparoscopic approach was found although the authors state that the analysis is underpowered. Similarly, in the two RCTs analyzed separately, there was no survival difference. The conclusive statement of the authors is that there is a survival benefit in favor of LLS and that laparoscopy is at least not inferior to the open approach.

This is the first high-quality meta-analysis providing encouraging findings of better survival following LLS and there is currently no superior data synthesis akin to this report. A recent meta-analysis published by Ciria et al., including only the OSLO-COMET trial, has shown better short-term results after LLS but no oncological advantages (9).

We support the cautious conclusion on superior survival after LLS as most of the included studies only report 3-year survival. But in line with the plurality of other reports on perioperative outcomes, confirming the superiority for LLS, this surely will further promote the already widespread use of the approach.

But cautiously we remind the reader that patient selection is an issue in propensity-score matched studies as it is in RCTs and hence may only conditionally be applied to the general population. We also believe that RCTs powered to detect a survival benefit may not be conducted due to the high number of patients needed to detect a survival difference.

Generally, the approach to surgical research is profoundly different from other medical fields. No strict regulations apply to testing and implementation of surgical techniques unlike for example the introduction of new pharmaceutical drugs. New drugs regularly must pass through multiple phases, from exploration to safety, benefit and comparison to the standard treatment before regulatory approval and widespread clinical use. In surgery, studies usually follow the clinical use. Therefore, the introduction of surgical techniques poses considerable methodological challenges and motivation to conduct high-quality RCTs may be low.
With the results from this and other high-quality data from meta-analyses, an evidence-based shift towards LLS as standard of care has started. More data from multicenter RCTs, the ORANGE II Plus trial comparing laparoscopic versus open hemihepatectomies (NCT00874224) and the ORANGE Segments trial comparing resections in the posterosuperior segments $(4 \mathrm{a}, 7,8)$ for the two approaches (NCT03270917) is expected.

We believe that a careful implementation, no matter how revolutionary a novel technique appears at first sight, is mandatory and doesn't hinder a steady progress. Multicenter randomized controlled trials should be conducted and international registries be initiated simultaneously. Analysis and reports from such collaborations should then be pooled in meta-analysis to help in the evaluation of the current practice and guide future research.

However, already now, there are good reasons to assume that LLS will become a standard approach for the treatment of CRLMs and may become a strong alternative to open liver surgery.

\section{Acknowledgments}

Funding: None.

\section{Footnote}

Conflicts of Interest: Both authors have completed the ICMJE uniform disclosure form (available at http://dx.doi. org/10.21037/hbsn.2020.04.03). The authors have no conflicts of interest to declare.

Ethical Statement: The authors are accountable for all aspects of the work in ensuring that questions related to the accuracy or integrity of any part of the work are appropriately investigated and resolved.

Open Access Statement: This is an Open Access article distributed in accordance with the Creative Commons Attribution-NonCommercial-NoDerivs 4.0 International License (CC BY-NC-ND 4.0), which permits the noncommercial replication and distribution of the article with the strict proviso that no changes or edits are made and the original work is properly cited (including links to both the formal publication through the relevant DOI and the license). See: https://creativecommons.org/licenses/by-nc-nd/4.0/. 


\section{References}

1. Cipriani F, Shelat VG, Rawashdeh M, et al. Laparoscopic parenchymal-sparing resections for nonperipheral liver lesions, the diamond technique: technical aspects, clinical outcomes, and oncologic efficiency. J Am Coll Surg 2015;221:265-72.

2. Berardi G, Igarashi K, Li CJ, et al. Parenchymal sparing anatomical liver resections with full laparoscopic approach: description of technique and short-term results. Ann Surg 2019. [Epub ahead of print].

3. van der Poel MJ, Barkhatov L, Fuks D, et al. Multicentre propensity score-matched study of laparoscopic versus open repeat liver resection for colorectal liver metastases. Br J Surg 2019;106:783-9.

4. Abu Hilal M, Aldrighetti L, Dagher I, et al. The southampton consensus guidelines for laparoscopic liver surgery: from indication to implementation. Ann Surg 2018;268:11-8.

5. Martínez-Cecilia D, Wicherts DA, Cipriani F, et al. Impact of resection margins for colorectal liver metastases

Cite this article as: Abu Hilal M, Kuemmerli C. Resections for colorectal liver metastasis: the breakthrough of laparoscopic surgery? HepatoBiliary Surg Nutr 2020;9(4):548-550. doi: 10.21037/hbsn.2020.04.03 in laparoscopic and open liver resection: a propensity score analysis. Surg Endosc 2020. [Epub ahead of print].

6. Fretland ÅA, Dagenborg VJ, Bjørnelv GMW, et al. Laparoscopic versus open resection for colorectal liver metastases: the OSLO-COMET randomized controlled trial. Ann Surg 2018;267:199-207.

7. Robles-Campos R, Lopez-Lopez V, Brusadin R, et al. Open versus minimally invasive liver surgery for colorectal liver metastases (LapOpHuva): a prospective randomized controlled trial. Surg Endosc 2019;33:3926-36.

8. Syn NL, Kabir T, Koh YX, et al. Survival advantage of laparoscopic versus open resection for colorectal liver metastases: a meta-analysis of individual patient data from randomized trials and propensity-score matched studies. Ann Surg 2019. [Epub ahead of print].

9. Ciria R, Ocaña S, Gomez-Luque I, et al. A systematic review and meta-analysis comparing the short- and longterm outcomes for laparoscopic and open liver resections for liver metastases from colorectal cancer. Surg Endosc 2020;34:349-60. 